\title{
Low-Angle X-Ray Spectroscopy and Reflectometry Techniques in Interdisciplinary Applications
}

\author{
A. Kubala-Kukuśs $\hat{S}^{a, b, *}$, D. Banaś ${ }^{a, b}$, M. PajeK ${ }^{a}$, J. Braziewicz $^{a, b}$, \\ S. GóźDź ${ }^{b, c}$, J. SzlachetKo ${ }^{d}$, J. Semaniak ${ }^{a}$, Ł. JABŁoński ${ }^{a}$, \\ P. Jagodziński ${ }^{a}$, M. PiWoWArczyK ${ }^{a}$, D. Sobota ${ }^{a}$, I. StabraWA ${ }^{a, b}$, \\ R. Stachura ${ }^{a}$, K. Szary ${ }^{a, b}$ And J. WudArCZYK-MoĆKO ${ }^{b}$ \\ ${ }^{a}$ Institute of Physics, Jan Kochanowski University, Uniwersytecka 7, 25-406 Kielce, Poland \\ ${ }^{b}$ Holy Cross Cancer Center, S. Artwińskiego 3, 25-734 Kielce, Poland \\ ${ }^{c}$ Institute of Public Health, Jan Kochanowski University, \\ IX Wieków Kielc 19, 25-317 Kielce, Poland \\ ${ }^{d}$ Institute of Nuclear Physics, Polish Academy of Sciences, \\ Radzikowskiego 152, 31-342 KrakĂłw, Poland
}

Doi: 10.12693/APhysPolA.139.247

*e-mail: aldona.kubala-kukus@ujk.edu.pl

\begin{abstract}
In this paper, the low-angle X-ray spectroscopy and reflectometry techniques, namely: the total reflection X-ray fluorescence analysis, grazing emission X-ray fluorescence analysis, grazing incidence X-ray fluorescence analysis, X-ray reflectometry and total reflection X-ray photoelectron spectroscopy, are presented. These techniques are used in the scientific activity, in interdisciplinary applications, at the Institute of Physics of the Jan Kochanowski University in Kielce (UJK) and the Department of Physical Methods of the Holy Cross Cancer Center (ŚCO). The studies were conducted in international cooperation with the European Synchrotron Radiation Facility (ESRF, Grenoble, France), the University of Fribourg (Switzerland) and Elettra Sincrotrone Trieste (Elettra, Trieste, Italy). The physical basics of the techniques are discussed as well as experimental setups applying either X-ray tube radiation or synchrotron radiation. Examples of the study possibilities and interdisciplinary applications, presented in the article, include elemental content analysis in human biological materials, biological and environmental samples (total reflection X-ray fluorescence analysis) and depth profiling of silicon wafer impurities (grazing emission X-ray fluorescence analysis). The paper also presents depth profiling, morphology study and surface elemental and chemical composition analysis of titanium and titanium dioxides nanolayers deposited on different substrates and irradiated by Xe ions in high charge states (grazing incidence X-ray fluorescence analysis, X-ray reflectometry, total reflection X-ray photoelectron spectroscopy).
\end{abstract}

topics: low-angle X-ray spectroscopy (TXRF, GEXRF, GIXRF), X-ray reflectometry (XRR), total reflection X-ray photoelectron spectroscopy (TRXPS)

\section{Introduction}

The analysis of physical processes accompanying the interaction of X-rays with matter, which are the physical bases of X-ray spectroscopy, gives unique possibilities to study various properties of the medium. Description of photoelectric absorption, elastic scattering, inelastic scattering or generally X-ray attenuation, in a different range of X-ray energy, is strongly related to the atomic properties of matter.

The study of the processes of interaction of $\mathrm{X}$-radiation with matter concentrates on the determination of characteristic quantities such as: crosssections, intensities of scattered and/or characteristic radiation induced in the photoelectric effect, attenuation coefficients or on the analysis of the secondary effects, such as the emission of photoelectrons and the Auger electrons. The obtained information about the properties of matter is very diverse, from the elemental composition and chemical composition, to information about the chemical environment of the selected atom, the densities of electron states or sample morphology.

Special information on atomic properties is provided by the analysis of the phenomenon of reflection and refraction of X-rays at the border of the two media [1]. In the case of X-rays, refraction index $n$ is smaller, by a small value $\delta$ (dispersion component), from unity and X-ray radiation propagates in the medium with the phase speed higher than the speed of light in vacuum. It means that 
for X-rays, matter is an optically less dense medium than vacuum $(n<1)$. The $\mathrm{X}$-ray radiation, going from vacuum to matter, refracts to the border of the medium. As a result, there exists a certain incidence critical angle $\phi=\phi_{c}$, above which there is total external reflection of X-rays. This phenomenon is the physical basis of many low-angle X-ray spectroscopy and reflectometry techniques. Usually, a glancing angle $\varphi$ is introduced, which is the angle between the incident $\mathrm{X}$-ray and the reflecting surface, defined as $\varphi=90^{\circ}-\phi$.

The phenomena of reflection and refraction of the X-ray beam at the border of the medium are described by three basic quantities characterizing these processes, namely: critical angle $\varphi_{c}$, reflection coefficient $R$ and penetration depth $z$ of X-ray.

Critical angle depends on the type of medium and is inversely proportional to energy $E$ of X-rays $\left(\varphi_{c} \sim \sqrt{\frac{Z \rho}{A}} \frac{1}{E}, Z\right.$ - atomic number, $A$ - mass number of the element medium, $\rho$ - density of the medium). For angles $\varphi<\varphi_{c}$, the reflection coefficient is practically the same for all materials, reaching the value of $R \approx 1$ (100\% of reflection). The penetration depth of X-rays into the medium under conditions of total reflection of X-rays reaches a constant value, on the order of a few nanometers [1].

Low-angle X-ray spectroscopy and reflectometry techniques are based on specific properties of the interaction of X-rays with matter in the angular ranges below and slightly above the critical angle. Atomic processes and properties of materials are studied under the conditions of total external reflection of primary excitation radiation and/or characteristic X-ray excited in a material, using a fixed angle of incidence/emission and/or also taking into account the dependence of the analyzed signals on the incidence/emission angle. The source of X-ray radiation can be both synchrotron radiation or, in laboratory conditions, an X-ray tube. Detection of the analyzed radiation takes place in a mode of the wavelength dispersion and/or energy dispersion, with high and/or low energy resolution. The unique aspect of application of X-ray total reflection is the effect of enhancing fluorescence intensity with a simultaneous decrease of the intensity of scattered radiation, which is the source of the background in the X-ray spectra determining the application possibilities of a given analytical technique (detection limit). Analogous aspects are discussed for nonradiative transitions, for which improving the ratio of photoelectron intensity to scattered electrons is observed.

This work presents a review of the low-angle $\mathrm{X}$-ray spectroscopy and reflectometry techniques applied in many years' scientific activity of the Department of Atomic Physics and Nanophysics of the Institute of Physics of the Jan Kochanowski University in Kielce (UJK) and the Department of Physical Methods of the Holy Cross Cancer Center (ŚCO) and also examples of interdisciplinary applications of these techniques. The studies were conducted in cooperation with the European Synchrotron Radiation Facility (ESRF, Grenoble, France), the University of Fribourg (Switzerland) and Elettra Sincrotrone Trieste (Elettra, Trieste, Italy).

In the paper, the following low-angle X-ray spectroscopy and reflectometry techniques are discussed: (a) total reflection X-ray fluorescence analysis (TXRF) [1], (b) grazing emission X-ray fluorescence analysis (GEXRF) [2], (c) grazing incidence X-ray fluorescence analysis (GIXRF) [1, 3] and X-ray reflectometry (XRR) $[4,5]$ and (d) total reflection X-ray photoelectron spectroscopy (TRXPS) [6-8]. In the paper, the physical basics, experimental setups, application possibilities and examples of interdisciplinary applications are presented.

\section{TXRF}

In the TXRF technique [1], the primary X-ray beam is directed on the studied sample (or on the sample carrier on which the sample is deposited) at an angle smaller than the critical angle, strictly defined for a given X-ray energy and type of sample/sample carrier material (see Fig. 1).

The main issue of the studied atomic processes is the registration of fluorescence radiation excited in photoelectric effect and primary radiation scattered elastically and inelastically (the Rayleigh and Compton effects), and the determination of its intensity, which gives information, also the quantitative one, about the elemental composition of the sample and the detection limit level. In the TXRF technique, the registration angle is $90^{\circ}$ (see Fig. 1). An important aspect is the selection of experimental conditions to maximize the emission probability of characteristic radiation and minimize the inelastic scattering of X-rays in the studied material.

The studies with the application of the TXRF technique were carried out using the TXRF system constructed in the Institute of Physics (based on a setup developed by a group from the Technical

\section{TXRF}

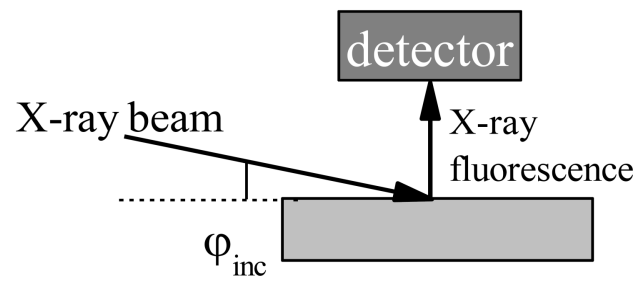

Fig. 1. Schematic presentation of the TXRF technique setup. In the TXRF technique, primary radiation is directed toward a sample (or on the sample carrier on which the sample is deposited) below the critical angle of the excitation radiation [9]. 
University in Vienna [10, 11]) and on the commercial spectrometer S2 Picofox (Bruker), in which the X-ray sources are X-ray tubes with a molybdenum anode (Mo). The maximum energy of bremsstrahlung radiation is 40 (45) $\mathrm{keV}$ (IF system) and $50 \mathrm{keV}$ (commercial spectrometer), respectively. Characteristic radiation used as the excitation radiation is $17.44 \mathrm{keV}$ (Mo- $K_{\alpha}$ line). The detectors applied for X-ray registration are: semiconductor $\mathrm{Si}(\mathrm{Li})$ (energy resolution $\approx 170 \mathrm{eV}$ for the $\mathrm{Fe}-K_{\alpha}$ line) and silicon drift detector (SDD, energy resolution $\approx 160 \mathrm{eV}$ for $\mathrm{Mn}-K_{\alpha}$ ), whereas the energy range of the registered radiation is from about $1.5 \mathrm{keV}$ to $18 \mathrm{keV}$. The critical angle for the applied excitation radiation $(17.44 \mathrm{keV})$ and the quartz sample carrier on which the analyzed samples are deposited is $0.1^{\circ}$.

In the applied TXRF experimental setup, the sample is generally assumed as a homogeneous thin target, for which additional effects accompanying the X-ray interaction with matter, typical for the classic XRF technique (for example matrix effect, secondary excitation, granulation), are negligibly small. This assumption is important in a correct interpretation of the characteristic radiation intensity emitted by a sample (calibration procedure of the experimental setup, additionally applying the internal standard).

This low-angle X-ray spectroscopy technique was used by our group for an elemental analysis of the following samples: water, wastewater, human biological materials (serum, hair, urine, blood components, tissues, kidney stones), plant and animal materials and food [12-18]. An example of the TXRF spectrum of a human serum sample excited by the primary X-rays generated in a Mo-anode

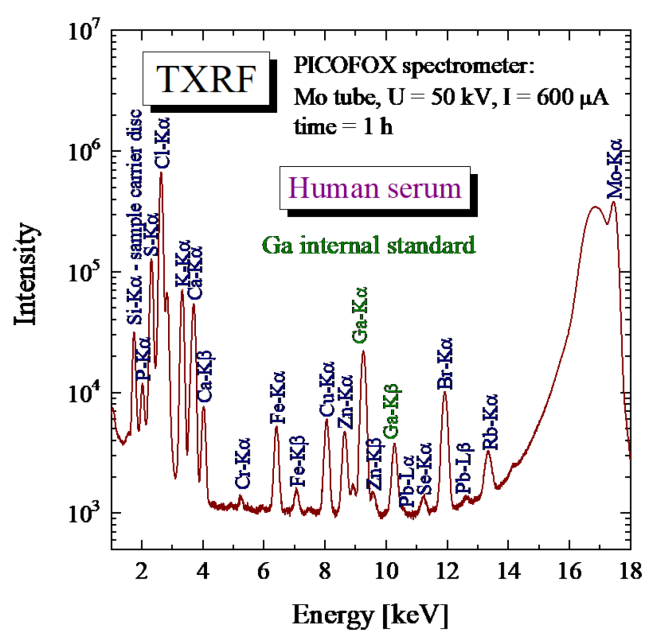

Fig. 2. An example of the TXRF spectrum of a human serum sample excited by the primary $\mathrm{X}$-rays generated in a Mo-anode X-ray tube operated with voltage $U=50 \mathrm{kV}$ and current $I=600 \mu \mathrm{A}$. The measurement time was one hour [14].
$\mathrm{X}$-ray tube is presented in Fig. 2. In general, the TXRF analysis allows to examine elements from magnesium (Mg) to uranium (U) (for technical reasons, except for the elements in the range from zirconium $(\mathrm{Zr})$ to ruthenium $(\mathrm{Ru}))$. The quantitative analysis of element concentration is based on the internal standard application (e.g., Ga, Y, Se) and spectrometer calibration. The detection limit for TXRF depends on the studied sample, sample preparation procedure and the analyzed element. The best value is achieved for a water sample and elements in the range from $\mathrm{Cr}$ to $\mathrm{Br}$, being on the level of $1 \mu \mathrm{g} / \mathrm{l}$.

The aim of the TXRF analysis was a routine analysis of trace element concentrations in human biological materials (e.g., $\mathrm{Cu}$, Se in human serum), determination of the correlation between the sample elemental composition and the patient disease state, determination of reference values of element concentration in human samples (serum, hair, urine), analysis of animal tissues after nanoparticles administration and monitoring of environmental pollution. The selection of research topics (samples), on the one hand, was closely related to the cooperation with the Holy Cross Cancer Center and, on the other hand, with great interest in biological, medical or environmental protection sciences in determining the content of elements in samples of human biological material.

\section{GEXRF}

The next technique of low-angle X-ray spectroscopy, discussed in the presented paper, is GEXRF $[1,2,19,20]$, similarly to the TXRF technique, based on the phenomenon of X-ray total reflection and specific aspects of X-ray interaction with matter in the range of critical angles. In the GEXRF technique, the primary X-ray beam is directed towards the studied sample at an angle of several tens of degrees (usually it is $90^{\circ}$, see Fig. 3).

Analogously to the TXRF technique, the main issue of the studied atomic processes is registering a characteristic X-ray excited in the sample atoms and then determining their intensity. A key aspect of the GEXRF technique is the fact that the emission angle, at which the fluorescence radiation is registered, is in the critical angle range. A critical angle value depends on characteristic radiation energy and on a sample or carrier type. Thus, the low-angle GEXRF technique focuses on the phenomenon of total reflection of the fluorescence radiation (and not, as in the TXRF technique, on primary excitation) (see Fig. 1). The determination of the emitted fluorescence radiation intensity in dependence on the emission angle (angular dependence) is a unique possibility when studying surface layer properties using the GEXRF technique.

The low-angle GEXRF technique was used by our group in studies presented in several articles $[9,21-23]$. The studies were carried out at the 


\section{GEXRF}

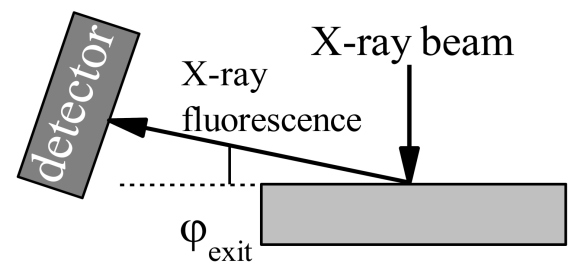

Fig. 3. Schematic presentation of the GEXRF technique setup. In the GEXRF method, below the critical angle, the emitted fluorescence X-rays are detected [9].

European Synchrotron Radiation Facility on the ID21 beamline (X-ray microscopy), in cooperation with the University of Fribourg, using a von Hamos crystal spectrometer with high energy resolution $(\approx 0.9 \mathrm{eV})[24]$.

First measurements [21] were dedicated to the study of the possibility to use the GEXRF technique with a high energy resolution in determining trace impurities of aluminum $(\mathrm{Al})$ on a silicon $(\mathrm{Si})$ surface. The goal of the study was to estimate the detection limit for $\mathrm{Al}$ impurities determination in a semiconductor material using the GEXRF technique in the context of the microelectronics development requirements (detection limit of less than $10^{10}$ at. $\left./ \mathrm{cm}^{2}[1]\right)$.

The limit of $\mathrm{Al}$ impurity detection by X-ray spectroscopy techniques, characterized by energy resolution above $100 \mathrm{eV}$, is, however, limited by the presence of resonance Raman scattering (RRS) [25]. In the case of the GEXRF technique, the effect of the special measurement geometry is a limited detection of $\mathrm{Si}-K_{\alpha}$ radiation, constituting the background for the fluorescence spectra of the studied impurities, reduced to a $\mathrm{Si}$ surface layer with a thickness of several $\mathrm{nm}$ and also a separation of the $\mathrm{Al}-K_{\alpha}$ line. A basic feature of the GEXRF method used in the studies of $\mathrm{Al}$ impurities in silicon is that, for the angles below the critical angle, a significant enhancement (of the order of $10^{3}$ ) of radiation intensity $\mathrm{Al}-K_{\alpha} / \mathrm{Si}-K_{\alpha}$ ratio is observed. In order to select optimal measurement conditions, the GEXRF $\mathrm{X}$-ray spectra of high resolution were modeled, assuming the synchrotron radiation excitation of a silicon wafer with $\mathrm{Al}$ impurities, using theoretical description [1] and the results of previous experiments carried out at the ESRF in which resonant Raman scattering in silicon was determined [26]. Based on calculations, the excitation energy was chosen as $1570 \mathrm{eV}$, for which the best detection limit of $\mathrm{Al}$ in silicon is obtained, resulting from good separation of the Al- $K_{\alpha}$ line and the RRS $K L$ profile (RRS where $K L$ represents the initial and final electron state).

In the studies described in [21], clean silicon was used (from the Institute of Electronic Materials in Warsaw) and silicon intentionally contaminated

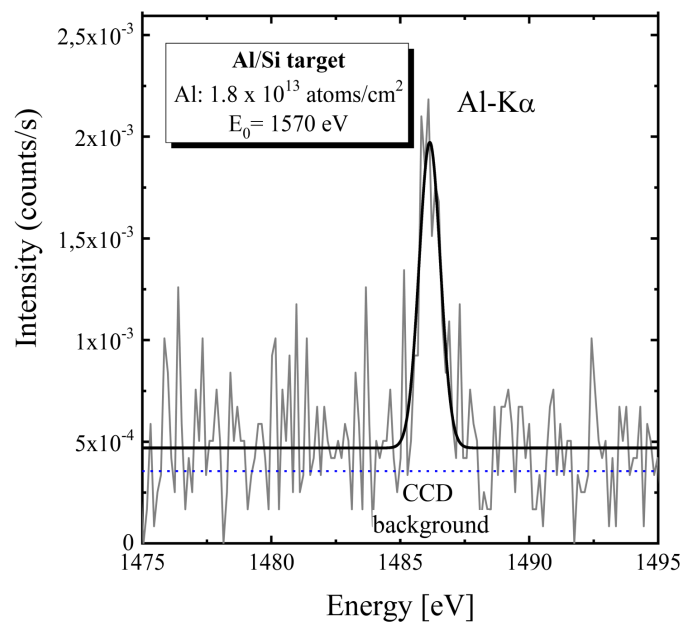

Fig. 4. High-resolution Al- $K_{\alpha}$ fluorescence X-ray line measured at a grazing emission angle of $\varphi=0.6^{\circ}$ for photon beam energy of $1570 \mathrm{eV}$ for a blank Si wafer with a $1.8 \times 10^{13} \mathrm{Al}$ at. $/ \mathrm{cm}^{2}$ surface contamination [21].

with $\mathrm{Al}$ atoms (dry residue of the reference solution $\mathrm{Al}\left(\mathrm{NO}_{3}\right)$ in $\mathrm{HNO}_{3}$ (Merck), $\mathrm{Al}$ atoms concentration $=3 \times 10^{14}$ at. $\left./ \mathrm{cm}^{2}\right)$.

Figure 4 presents fluorescence $\mathrm{Al}-K_{\alpha}$ line, registered with high energy resolution for emission angle $\varphi=0.6^{\circ}$ and synchrotron radiation with energy $1570 \mathrm{eV}$ (intensity $\approx 10^{11}$ photons/s), emitted from silicon. The level of surface $\mathrm{Al}$ impurities was estimated at the level of $1.8 \times 10^{13} \mathrm{Al}$ at. $/ \mathrm{cm}^{2}$ [21]. In the experiment, the detection limit for $\mathrm{Al}$ in $\mathrm{Si}$ was achieved as $10^{12}$ at. $/ \mathrm{cm}^{2}$ which, while applying an additional chemical preconcentration method (VPD) [1], corresponds to $10^{7}$ at. $/ \mathrm{cm}^{2}$.

In [21], it was presented that the low-angle GEXRF technique can be used to determine not only surface contamination but also a depth distribution of impurities, on a scale of nano- and micrometers, respectively.

The depth distribution of the studied element is obtained from the experimentally determined intensity dependence of the emitted fluorescent radiation on the emission angle. The dependence shape varies with the morphology and surface layer thickness. In the performed experiments, the angular dependence for various surface structures was determined (thick $\mathrm{Al}$ substrate, thick $\mathrm{Si}$ substrate, thin $\mathrm{Al}$ film deposited on $\mathrm{Si}$ ) (see Fig. 5). In the case of a thin $\mathrm{Al}$ layer on $\mathrm{Si}$, the experimental dependence is well described by theoretical calculations, in particular below the critical angle, assuming that the deposited Al layer is not homogeneous but has an island-like structure, with the height of $2.2 \mathrm{~nm}$ in the discussed case. Forming islands during the formation of thin layers, in particular $\mathrm{Al}$ on $\mathrm{Si}$, is a known VolmerWeber process $[27,28]$.

Research on semiconductor materials using the GEXRF technique with high energy resolution and with the excitation using synchrotron radiation was 


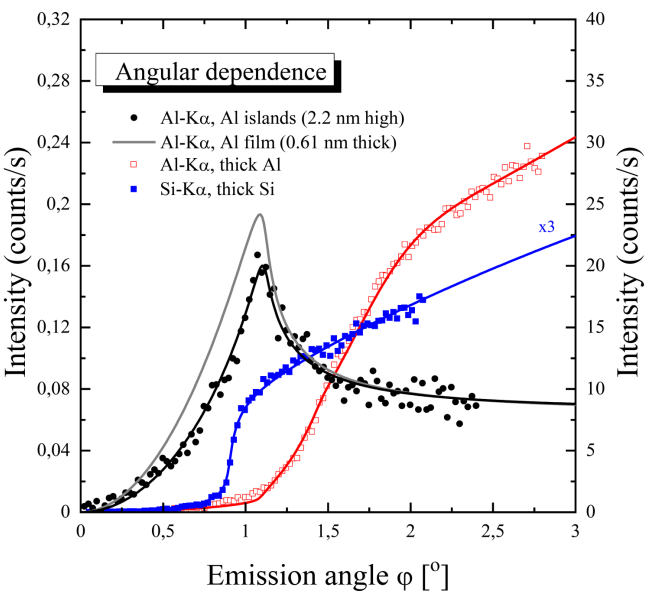

Fig. 5. Measured intensities of the Al- $K_{\alpha}$ fluorescence line excited by $1570 \mathrm{eV}$ photons versus the grazing emission angle for a thin $\mathrm{Al}$ layer on a $\mathrm{Si}$ wafer and a thick Al sample. Angular dependence of the $K_{\alpha}$ fluorescence line of a thick Si sample bombarded by $2000 \mathrm{eV}$ photons is also shown. Solid lines are the result of calculations [29] performed for filmlike (0.61 nm thick) and island-like (2.2 nm high) $\mathrm{Al}$ layers (left scale), and thick $\mathrm{Al}_{2} \mathrm{O}_{3} / \mathrm{Al}$ and $\mathrm{Si}$ samples (right scale) [21].

reported in [22]. The aim of the research was to determine the detection limit of the GEXRF method for various elements which are surface impurities on silicon wafers.

In the experiment performed at the ESRF, the synchrotron radiation with $3.65 \mathrm{keV}$ energy was used to excite fluorescence lines of the following elements: Y, Tl, Pb, Bi, Ag, Cd, K and $7.2 \mathrm{keV}$ for elements: $\mathrm{Ca}, \mathrm{Ba}, \mathrm{Ti}, \mathrm{Cr}, \mathrm{Mn}, \mathrm{Fe}$. Series $K_{\alpha}, L_{\alpha}$ and $M_{\alpha}$ of fluorescence radiation as regards various elements in the energy range from $2 \mathrm{keV}$ to $7 \mathrm{keV}$ were analyzed. The X-ray spectra were registered for emission angles of $0.2^{\circ}, 0.6^{\circ}$, and $0.7^{\circ}$, depending on the energy of the analyzed fluorescent radiation, fulfilling the condition of total X-ray reflection.

Based on the registered spectra, the detection limit (the ratio of scattered radiation to fluorescent radiation) of the analyzed elements was determined (see Fig. 6), which for direct GEXRF measurements is of the order of $10^{12}$ at. $/ \mathrm{cm}^{2}$. The obtained value is comparable and for some elements even smaller than the detection limit of the TXRF method, normally used in the control process of contamination concerning semiconductor materials. By combining the GEXRF technique with additional preconcetration chemical methods (VPD), it is possible to obtain elements detection at $10^{7}$ at. $/ \mathrm{cm}^{2}$ [30].

The low-angle GEXRF technique was also used to analyze the depth profiles of trace impurities of semiconductor materials with light elements [23]. In the studies, the intensity of $\mathrm{Al}-K_{\alpha}$ radiation emitted by aluminum atoms implanted in silicon [23] depending on the emission angle in the range of the critical angle of $\mathrm{Al}-K_{\alpha}$ radiation was

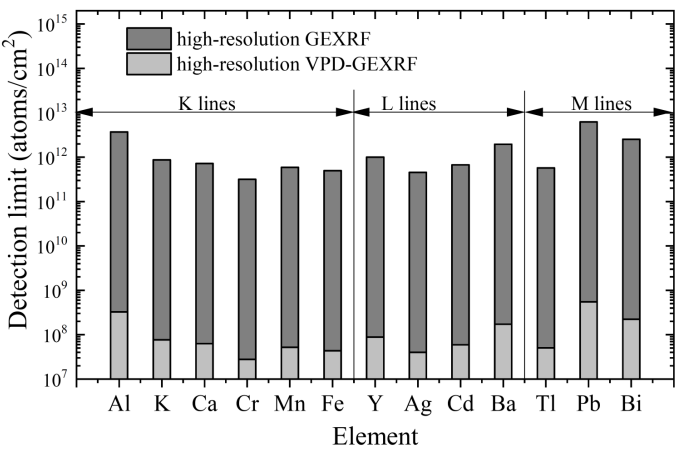

Fig. 6. Detection limit for the GEXRF technique [22].

registered.

Due to the fact that the shape of depth profiles is determined by the energy of the implanted ions and ion concentration by the implantation dose, the measurements were performed for silicon samples (Si (100), thickness of $0.2 \mathrm{~mm}$ ) implanted by $\mathrm{Al}$ ions of different energies: 1, 5, 10, 15, 20, 25, 30, 50 and $100 \mathrm{keV}$ and implant dose of $10^{16} \mathrm{ions} / \mathrm{cm}^{2}$. The GEXRF measurements were made for $1.582 \mathrm{keV}$ synchrotron radiation energy (to excite the emission of the $\mathrm{Al} K_{\alpha}$ line) and for $2.0 \mathrm{keV}$ (for Si $K_{\alpha}$ line) using the von Hamos spectrometer.

From the angular dependence of the intensity of fluorescent radiation, depth profiles of $\mathrm{Al}$ ions in silicon (Gaussian distributions) were reconstructed and the distribution parameters (expected value, standard deviation) were estimated, with a very good agreement between experimental results and theoretical calculations obtained [23].

The presented GEXRF studies initiated a cycle of articles related to the analysis of the emission of the fluorescence radiation under conditions of total $\mathrm{X}$-ray reflection for various models of nanolayers, using the low-angle GEXRF technique with a high energy resolution [31-34].

\section{GIXRF and XRR}

Additional analytical capabilities of the low-angle TXRF technique, which gives information on the sample elemental composition, can be obtained if the possibility of changing the angle of incidence is additionally provided. In this technique, called the GIXRF [1], the primary X-ray beam is directed on the sample at an angle in the range of the critical angle, and for a varying glancing angle the fluorescence radiation emitted from the sample is registered. The angular dependence of the characteristic radiation intensity gives information of the sample depth profile. Many experimental systems of the GIXRF technique also enable the simultaneous measurement of the primary X-ray beam elastically scattered on samples as a function of a grazing (incidence) angle $[4,5]$ which is a physical basis of $\mathrm{XRR}$. The XRR configuration is used to study thin 


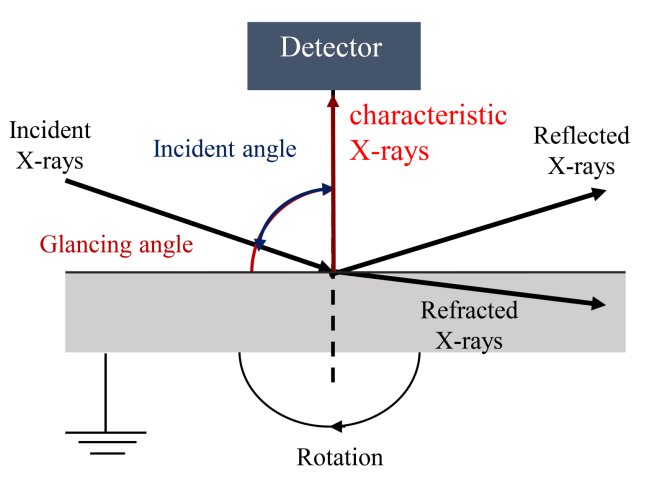

Fig. 7. Scheme of the GIXRF and XRR techniques.

layers by analyzing the intensity changes of the reflected beam in the low-angle range. These changes are the results of specular reflection of X-rays from different interfaces (e.g., substrate-layer and layerair or layer-layer) of a sample and further interference of the reflected X-ray. The XRR technique provides information on the thickness, density and roughness of the studied thin (1-500 nm) layer. Figure 7 presents the scheme of the GIXRF and XRR techniques.

The GIXRF and XRR were used by our group in the investigation of the $\mathrm{Ti}$ and $\mathrm{TiO}_{2}$ nanolayers with different thickness and deposited on different substrates, namely: the thickness of 25, 50 and $75 \mathrm{~nm}$ deposited on silicon (Si, (111)) or quartz $\left(\mathrm{SiO}_{2},(101)\right)$ or glass BK7 substrates, before and after an implantation with Xe ions in different (high and low) charge states $[35,36]$. The nanolayers irradiation was performed at the Kielce EBIS facility [37]. For calibration reasons, also bulk Ti and Si samples were studied.

In the GIXRF and XRR studies, the synchrotron radiation excitation was applied and these measurements were carried out at Elettra, at the X-ray fluorescence beamline. In the experiment, the monochromatized $\mathrm{X}$-ray beam of a resolving power $5 \times 10^{-3}$, size of $200 \mu \mathrm{m} \times 100 \mu \mathrm{m}$, an angular divergence of $0.15 \mathrm{mrad}$ and the photon flux about $2.5 \times 10^{11}$ photons/s was applied. The sample ultrahigh vacuum chamber is equipped with three motorized stages (for alignment of the chamber with respect to the synchrotron beam) and a seven-axis motorized manipulator for positioning the sample. In the setup, two SDDs for GIXRF and XRR measurements and three Si photodiodes as X-ray monitoring detectors were applied. The studied samples were irradiated by the X-ray beam of $6.0 \mathrm{keV}$ energy, for which the critical angle is $0.3^{\circ}$ for $\mathrm{Si}$ and $0.389^{\circ}$ for $\mathrm{TiO}_{2}$.

The XRR analysis was also performed in the Institute of Physics of the Jan Kochanowski University using an X'Pert Pro MPD diffractometer (Panalytical) with the X-ray excitation of $\mathrm{Cu}-K_{\alpha}$ line energy from an X-ray tube. The scattered radiation was registered using a silicon strip detector [35].

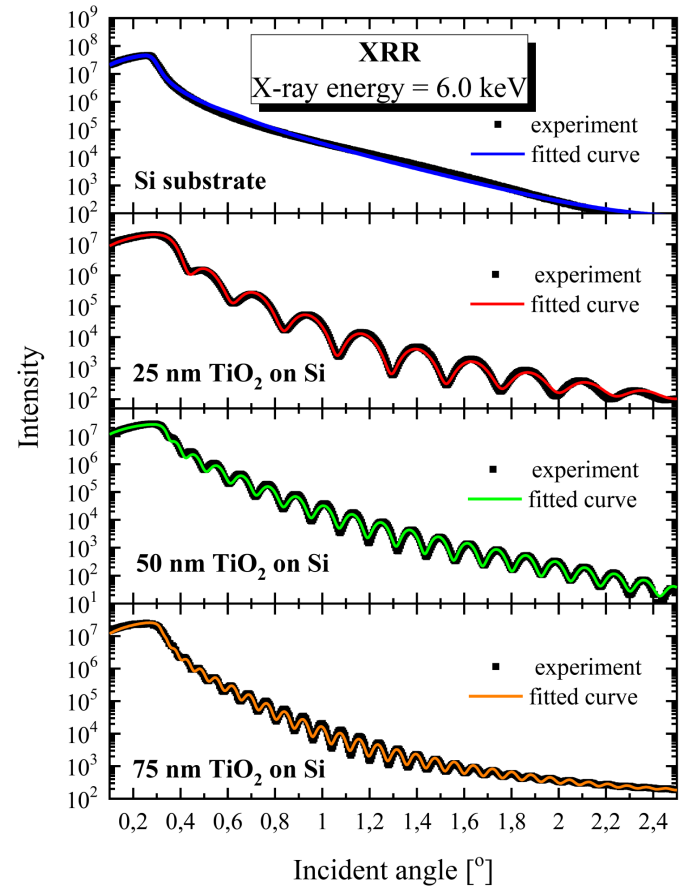

Fig. 8. Reflectivity patterns (black points) measured for the bulk Si (substrate) and 25, 50, and $75 \mathrm{~nm}$ titanium dioxide layers deposited on the $\mathrm{Si}$ substrate compared with theoretical patterns modelled and fitted using X'Pert Reflectivity 1.3a software (PANalytical) [36].

The examples of experimental reflectometric curves (black points) measured using synchrotron radiation for the bulk $\mathrm{Si}$ (substrate) and $\mathrm{TiO}_{2}$ nanolayers of 25, 50 and $75 \mathrm{~nm}$ thickness deposited on a $\mathrm{Si}$ substrate are shown in Fig. 8. The experimental curves are compared with theoretical patterns modeled and fitted using X'Pert Reflectivity 1.3a software (PANalytical) from which the density, thickness and roughness of the studied nanolayers were estimated $[35,36]$.

In the case of implanted nanolayers, the differences in the GIXRF and XRR curves were observed in dependence of the Xe ions charge states, wherein the biggest change was observed for $\mathrm{Xe}^{35+}$ ions (see Fig. 9 for GIXRF curves). It was observed that irradiation changes the roughness of the top amorphic $\mathrm{TiO}_{2}$ layer from $1.5 \mathrm{~nm}$ (for non-modified) to $1 \mathrm{~nm}$ (for irradiated by $\mathrm{Xe}^{35+}$ ) (see Fig. 10).

\section{TRXPS}

In the above-mentioned studies of atomic processes under excitation/emission conditions in the range of critical angles, the exciting factor was X-rays (primary), while the photoelectric effect and the emission and detection of the characteristic X-ray radiation were the analyzed processes (TXRF, GEXRF) [1]. Unique information about the properties of matter (electron binding energy, elemental composition of surface layers in the nanometer depth range, chemical environmental 


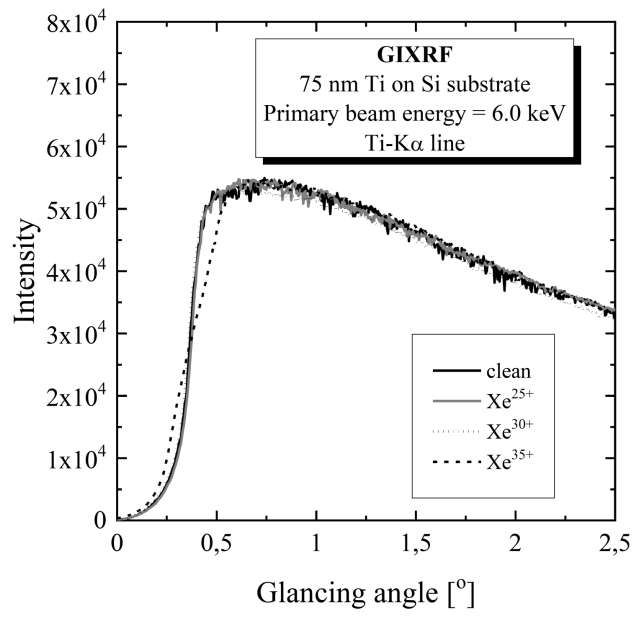

Fig. 9. GIXRF angular dependence of the Ti- $K_{\alpha}$ line intensity measured for $75 \mathrm{~nm} \mathrm{Ti}$ nanolayers deposited on Si substrate, clean and irradiated by $\mathrm{Xe}^{25+}, \mathrm{Xe}^{30+}$ and $\mathrm{Xe}^{35+}$ ions.

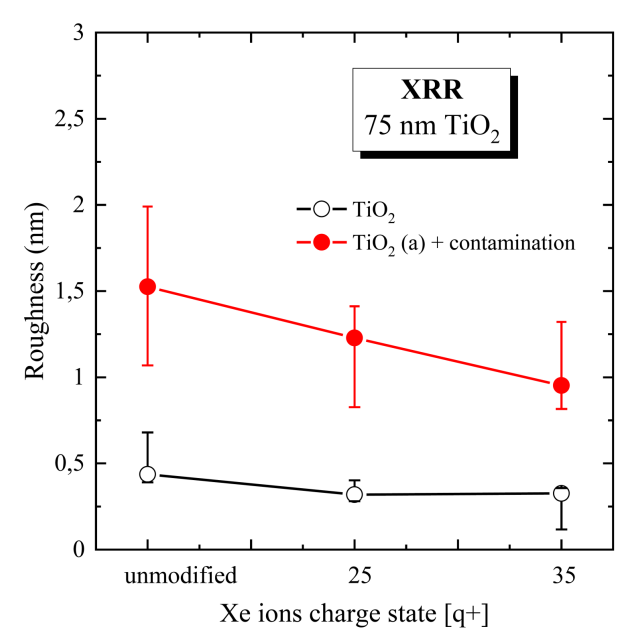

Fig. 10. Comparison of the roughness of the $\mathrm{TiO}_{2}$ nanolayer, non-modified and irradiated with highly charged Xe ions with charge state $25^{+}$and $35^{+}$. The presented results are for two layers according to the assumed sample model (substrate $(\mathrm{Si})$, main $\mathrm{TiO}_{2}$ layer and top layer $\mathrm{TiO}_{2}(\mathrm{a})+$ contaminations) [36]

of the element) provides also photoelectron detection, which is the physical basis of XPS [38-40]. In the classic XPS technique, a low energy $(1.5-3 \mathrm{keV})$ $\mathrm{X}$-ray beam is directed towards the studied matter at an angle of several tens of degrees and the detection limit of this technique is at the order of $0.1 \div 1$ at. $\%$. Analogously to the previously discussed low-angle X-ray spectroscopy (TXRF, GEXRF), an improvement in the detection limit can be obtained in the XPS technique using low-angle X-ray excitation (in the critical angle range) $[7,8]$. Investigations of the atomic properties with photoelectron spectroscopy in the range of X-rays with the total reflection of the incident beam (TRXPS) are presented in [41]. The TRXPS scheme is shown in Fig. 11.

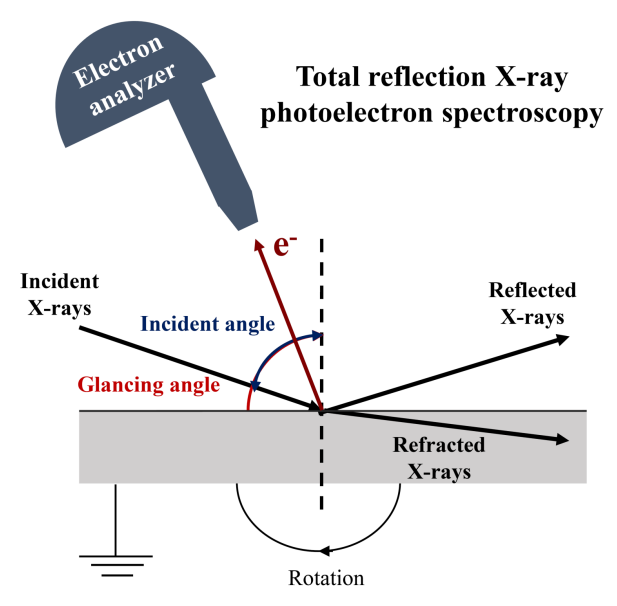

Fig. 11. Scheme of the TRXPS technique [41].

The glancing angle between the X-ray beam and the surface of the sample is lower (or very close) than the critical angle for a given energy and the material being studied.

Under such geometrical conditions, X-rays do not penetrate deeper into the sample than the range of the evanescent wave (on the order of $20-40 \AA$, depending on the type of material, radiation energy and glancing angle). Primary radiation intensity in this range can be up to four times higher than the intensity of the primary beam in classic conditions due to the effect of forming a standing wave on the surface layer of the sample. As a consequence, the emission of photoelectrons increases. The application of such a measurement geometry results in a background decrease in the spectra of photoelectrons, also due to a smaller inelastic scattering of photoelectrons.

The XPS technique, both in the classical and lowangle excitation, was used in the study of nanolayers $(25 \mathrm{~nm}, 75 \mathrm{~nm})$ of titanium (Ti) and titanium dioxide $\left(\mathrm{TiO}_{2}\right)$ deposited on $\mathrm{Si}(111)$ and $\mathrm{SiO}_{2}(101)$ substrates, before and after implantation by Xe ions in high charge states $\left(\mathrm{Xe}^{35+}, 280 \mathrm{keV}\right)$. In general, the study of surface modifications of titanium and titanium alloys is important in biomedical applications due to the use of these materials in biomedical devices and components $[42,43]$. The production of nanostructures on metallic, semiconductor and molecular materials also has huge importance in many fields of science, for example to develop new technologies for the production of small functional electronic systems with nanometer dimensions [44].

The XPS/TRXPS measurements [41] were performed at the Institute of Physics of the Jan Kochanowski University, using the XPS system (SPECS), with the excitation by monochromatic $\mathrm{Al}-K_{\alpha}$ radiation $(1486.7 \mathrm{eV})$ generated in an $300 \mathrm{~W}$ $\mathrm{X}$-ray tube. The primary X-ray was monochromatized using a FOCUS 500 quartz single crystal mirror with $500 \mathrm{~mm}$ Rowland circle and rocking curve width $\approx 160 \mathrm{meV}$. The XPS/TRXPS spectra were registered using a PHOIBOS 100 

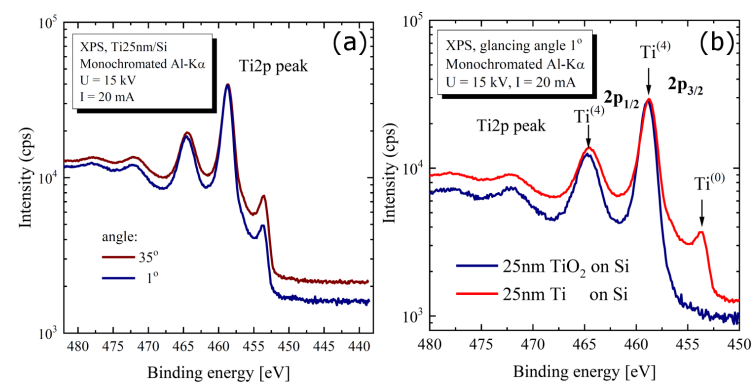

Fig. 12. (a) Spectrum of the photoelectrons in the binding energy range of Ti $2 p(450-480 \mathrm{eV})$ emitted from the Ti layer with the thickness of $25 \mathrm{~nm}$ deposited on $\mathrm{Si}$, for incidence angles $35^{\circ}$ and $1^{\circ}$ (X-ray total external reflection). (b) Comparison of the photoelectron spectrum in the energy range of $\mathrm{Ti} 2 p$ emitted from the layers $\mathrm{Ti}$ and $\mathrm{TiO}_{2}$ with the thickness of $25 \mathrm{~nm}$ deposited on $\mathrm{Si}$, for glancing angle $1^{\circ}$. In the figure, the photoelectron peaks for different $\mathrm{Ti}$ oxidation levels both for $\mathrm{Ti} 2 p_{1 / 2}$ and Ti $2 p_{3 / 2}$ states are marked [41].

electron analyzer with a one-dimensional (1D) delay line detector (DLD), under high vacuum conditions $\left(\approx 10^{-9} \mathrm{mbar}\right)$. For charge compensation, a flood gun FG-500 was applied. The value of FWHM for the $\mathrm{Ag} 3 d_{3 / 2}$ calibration photoelectron line was $1 \mathrm{eV}$.

Sample irradiation with $\mathrm{Xe}^{35+}$ ions (ion energy $8 \mathrm{keV} \times q=280 \mathrm{keV}$ ( $q$ is ons charge state), implantation dose $\approx 10^{10}$ ions $/ \mathrm{cm}^{2}$ ) was carried out at the Institute of Physics of the Jan Kochanowski University using an EBIS accelerator [37].

The XPS and TRXPS measurements were performed for the following excitation angles: $35^{\circ}, 25^{\circ}$, $15^{\circ}, 5^{\circ}$ and $1^{\circ}$ (below the critical angle, which for the energy of excitation $1486.7 \mathrm{eV}$, is $1.6^{\circ}(\mathrm{Ti})$ and $\left.1.59^{\circ}\left(\mathrm{TiO}_{2}\right)\right)$ and the survey spectra were registered in the binding energy range from 0 to $1400 \mathrm{eV}$. The following photoelectron peaks were identified: $\mathrm{O} 1 s$, Ti $2 p, \mathrm{~N} 1 s, \mathrm{C} 1 s, \mathrm{~B} 1 s$, Ti $3 s$, Ti $3 p$ and the Auger electrons: C KLL, N KLL, Ti $L M M$, $\mathrm{O} K L L$. The detailed spectra were also detected in the binding energy as regards the following photoelectrons: C $1 s, \mathrm{~N} 1 s$, Ti $2 p$ (for example Fig. 12a), $\mathrm{Ti} 3 s$, $\mathrm{O} 1 s$, $\mathrm{Xe} 3 d$, and $\mathrm{Xe} 4 d$ (for nanolayers irradiated with $\mathrm{Xe}$ ions). Next, the analysis of the recorded spectra was performed. In order to determine the detection limit of the TRXPS technique in a wide range of binding energy, the intensity of the photoelectron peak components and the background level in the spectra depending on the glancing angle value were analyzed.

Exemplary analysis for the range of binding energy of Ti $2 p_{1 / 2}$ and $\mathrm{Ti} 2 p_{3 / 2}$ electrons, for different oxidation states of $\mathrm{Ti}$, is shown in Fig. 12b [45]. It was obtained that excitation under X-ray total reflection condition improves the detection limit (LLD) about two times (for example, for the component $\operatorname{Ti} 2 p_{3 / 2}(0)$, it is 0.560 at. $\%$ for angle $35^{\circ}$ and 0.234 at. $\%$ for angle $1^{\circ}$ ). For the $25 \mathrm{~nm} \mathrm{Ti}$ nanolayer, the thickness of the oxidized layer was estimated as $3.052 \pm 0.037 \mathrm{~nm}$, based on the determined mean electron free path [46].

The influence of nanolayers implantation of $\mathrm{Xe}^{35+}$ ions was also analyzed in the studies. First, the Xe ion distribution was simulated in a two-layer system $\left(25 \mathrm{~nm} \mathrm{Ti}\right.$ layer on $\mathrm{Si}$ and $25 \mathrm{~nm} \mathrm{TiO}_{2}$ layer on Si substrate) for the ion energy of $280 \mathrm{keV}$, using the SRIM software $[47,48]$. In the case of Ti material, the range and straggle of the ion distribution are $103.0 \mathrm{~nm}$ and $38.2 \mathrm{~nm}$ and for $\mathrm{TiO}_{2}$ material they are $118.8 \mathrm{~nm}$ and $33.5 \mathrm{~nm}$, respectively. Due to relatively high values of the ion range (compared to depths analyzed in the XPS technique), Xe detection is at the level of the detection limit as regards XPS. On analyzing the photoelectrons $\mathrm{O} 1 s, \mathrm{C} 1 s$ and Ti $2 p$ lines, however, changes in the intensity of the components of each line were observed before and after Xe irradiation.

\section{Conclusions}

In this paper, the low-angle $\mathrm{X}$ spectroscopy and reflectometry techniques, TXRF, GEXRF, GIXRF, $\mathrm{XRR}$ and TRXPS, have been presented in the context of interdisciplinary studies conducted by the Atomic Physics and Nanophysics Department of the Institute of Physics of the Jan Kochanowski University in Kielce. The common feature of these techniques is the application of the X-ray total external reflection. Different experimental setups and measurement conditions were discussed, based both on $\mathrm{X}$-ray tube excitation and on synchrotron radiation, high and low-energy resolution of secondary radiation detection.

The TXRF technique was presented as the method useful in elemental composition analysis of different samples (e.g., biological, human biological material, environmental material) on the $\mathrm{ng} / \mathrm{g}$ detection limit level. The GEXRF technique can give information about sample morphology, depth profiling and surface impurities and the unique property of the applied X-ray detection system (von Hamos crystalline spectrometer) was high-energy resolution on the $1 \mathrm{eV}$ level. The combined GIXRF and XRR techniques allow to investigate sample morphology (thickness, density and roughness) in the tens of nanometers scale, as was discussed for $\mathrm{Ti}$ and $\mathrm{TiO}_{2}$ nanolayers deposited for different substrates and irradiated by $\mathrm{Xe}$ ions in high charge states. Finally, the TRXPS technique, as a classic XPS, gives information about the surface elemental composition and elements' chemical environment, in the range up to about $10 \mathrm{~nm}$, but ensures a better detection limit due to the sample excitation in the primary X-ray total external reflection regime.

The presented low-angle X-ray spectroscopy and reflectometry techniques can be successfully used in the study of various material properties in different interdisciplinary studies. 


\section{Acknowledgments}

The equipment was purchased thanks to the financial support of the European Regional Development Fund under the framework of the Polish Innovative Economy Operational Program (contract No. WNP-POIG.02.02.00-26-023/08) and the Development of Eastern Poland Program (contract No. POPW.01.01.00-26-013/09-04).

This work has been supported by the National Science Centre, Poland, under grant No. DEC2017/01/X/ST2/01913.

The authors would also like to acknowledge the contribution of the COST ACTION CA18130.

\section{References}

[1] R. Klockenkämper, A. von Bohlen, TotalReflection X-ray Fluorescence Analysis and Related Methods, Wiley, Hoboken (NJ) 2015.

[2] H.P. Urbach, P.K. de Bokx, Phys. Rev. B 53, 3752 (1996).

[3] A. von Bohlen, Spectrochim. Acta B 64, 821 (2009).

[4] Applications and Developments of Thin Film Technology, Handbook of Thin-Film Technology, Eds. H. Frey, H.R. Khan, Springer, 2015.

[5] V. Holy, U. Pietsch, T. Baumbach, HighResolution X-Ray Scattering from Thin Films and Multilayers, Springer Tracts in Modern Physics, Springer, 1999.

[6] J. Kawai, M. Takami, M. Fujinami, Y. Hashiguchi, S. Hayakawa, Y. Gohshi, Spectrochim. Acta B 47, 983 (1992).

[7] J. Kawai, J. Electron. Spectrosc. Relat. Phenom. 178-179, 268 (2010).

[8] A. Alshehabi, N. Sasaki, J. Kawai, Spectrochim. Acta B 114, 34 (2015).

[9] A. Kubala-Kukuś, D. Banaś, M. Pajek, J. Szlachetko, P. Jagodziński, J. Susini, M. Salomé, Radiat. Phys. Chem. 93, 117 (2013).

[10] P. Wobrauschek, P. Kregsamer, Spectrochim. Acta B 44, 453 (1989).

[11] P. Wobrauschek, P. Kregsamer, C. Streli, H. Aiginger, X-Ray Spectrom. 20, 23 (1991).

[12] A. Kubala-Kukuś, D. Banaś, J. Braziewicz, S. Góźdź, U. Majewska, M. Pajek, Spectrochim. Acta B 62, 695 (2007).

[13] A. Kubala-Kukuś, D. Banaś, J. Braziewicz et al., Biol. Trace Elem. Res. 158, 22 (2014).

[14] A. Kubala-Kukuś, D. Banaś, U. Majewska, I. Stabrawa, J. Wudarczyk-Moćko, S. Góźdź, X-Ray Spectrom. 45, 318 (2016).
[15] A. Kubala-Kukuś, M. Arabski, I. Stabrawa et al., X-Ray Spectrom. 46, 412 (2017).

[16] T. Magalhães, M.L. Carvalho, A. von Bohlen, M. Becker, Spectrochim. Acta B 65, 493 (2010).

[17] H. Stosnach, M. Mages, Spectrochim. Acta $B$ 64, 354 (2009).

[18] M. Arabski, I. Stabrawa, A. Kubala-Kukuś et al., Eur. Biophys. J. 48, 111 (2019).

[19] R.S. Becker, J.A. Golovchenko, J.R. Patel, Phys. Rev. Lett. 50, 153 (1983).

[20] Y. Yoneda, T. Horiuchi, Rev. Sci. Instrum. 42, 1069 (1971).

[21] A. Kubala-Kukuś, D. Banaś, W. Cao et al., Phys. Rev. B 80, 113305 (2009).

[22] J. Szlachetko, D. Banaś, A. Kubala-Kukuś et al., J. Appl. Phys. 105, 086101 (2009).

[23] Y. Kayser, D. Banaś, W. Cao et al., Spectrochim. Acta B 65, 445 (2010).

[24] J. Hoszowska, J.-Cl. Dousse, J. Kern, Ch. Rhême, Nucl. Instrum. Methods Phys. Res. A 376, 129 (1996).

[25] A. Kotani, S. Shin, Rev. Mod. Phys. 73, 203 (2001).

[26] J. Szlachetko, J.-Cl. Dousse, J. Hoszowska, M. Pajek, R. Barrett, M. Berset, K. Fennane, A. Kubala-Kukus, M. Szlachetko, Phys. Rev. Lett. 97, 073001 (2006).

[27] Z. Gai, B. Wu, J.P. Pierce, G.A. Farnan, D. Shu, M. Wang, Z. Zhang, J. Shen, Phys. Rev. Lett. 89, 235502 (2002).

[28] N. Joshi, A.K. Debnath, D. Aswal, K.P. Muthe, M.S. Kumar, S.K. Gupta, J.V. Yakhmi, Vacuum 79, 178 (2005).

[29] H.P. Urbach, P.K. de Bokx, Phys. Rev. B 63, 085408 (2001).

[30] D. Hellin, S. De Gendt, N. Valckx, P.W. Mertens, C. Vincker, Spectrochim. Acta B 61, 496 (2006).

[31] Y. Kayser, P. Hönicke, D. Banaś, J.-Cl. Dousse, J. Hoszowska, P. Jagodziński, A. Kubala-Kukuś, S.H. Nowak, M. Pajek, J. Anal. At. Spectrom. 30, 1086 (2015).

[32] S.H. Nowak, D. Banaś, W. Błachucki et al., Spectrochim. Acta B 98, 65 (2014).

[33] Y. Kayser, J. Szlachetko, D. Banaś, W. Cao, J.-Cl. Dousse, J. Hoszowska, A. Kubala-Kukuś, M. Pajek, Spectrochim. Acta B 88, 136 (2013).

[34] Y. Kayser, D. Banaś, W. Cao et al., X-Ray Spectrom. 41, 98 (2012).

[35] I. Stabrawa, A. Kubala-Kukuś, D. Banaś, G. Pepponi, J. Braziewicz, M. Pajek, M. Teodorczyk, Thin Solid Films 671, 103 (2019). 
[36] R. Stachura, A. Kubala-Kukuś, D. Banaś et al., Acta Phys. Pol. A 137, 38 (2020).

[37] D. Banaś, Ł. Jabłoński, P. Jagodziński, A. Kubala-Kukuś, D. Sobota, M. Pajek, Nucl. Instrum. Methods Phys. Res. B 354, 125 (2015).

[38] P. van der Heide, X-ray Photoelectron Spectroscopy: An Introduction to Principles and Practices, Wiley, 2011.

[39] C. Brundle, G. Conti, P. Mack, J. Electron. Spectrosc. Relat. Phenom. 178-179, 433 (2010).

[40] D. Baer, M. Engelhard, J. Electron. Spectrosc. Relat. Phenom. 178-179, 415 (2010).

[41] A. Kubala-Kukuś, D. Banaś, I. Stabrawa, K. Szary, D. Sobota, U. Majewska, J. Wudarczyk-Moćko, J. Braziewicz, M. Pajek, Spectrochim. Acta Part B 145, 43 (2018).

[42] X. Liu, P. Chu, C. Ding, Mater. Sci. Eng. $R$ 47, 49 (2004).
[43] T. Yoshida, S. Niimi, M. Yamamoto, S. Ogawa, T. Nomoto, S. Yagi, Nucl. Instrum. Methods Phys. Res. B 365, 79 (2015).

[44] I. Stabrawa, D. Banaś, A. Kubala-Kukuś et al., Nucl. Instrum. Methods Phys. Res. $B$ 408, 235 (2017).

[45] M.C. Biesinger, L.W.M. Lau, A.R. Gerson, R.St.C. Smart, Appl. Surf. Sci. 257, 887 (2010).

[46] H. Shinotsuka, S. Tanuma, C.J. Powell, D.R. Penn, Surf. Interf. Anal. 47, 871 (2015).

[47] J. Ziegler, M. Ziegler, J. Biersack, Nucl. Instrum. Methods Phys. Res. B 268, 1818 (2010).

[48] The Stopping and Range of Ions in Matter (SRIM), 2010, www.srim.org. 